\title{
SECURITY EVALUATION OF INFORMED WATERMARKING SCHEMES
}

\author{
Sofiane BRACI, Rémy BOYER and Claude DELPHA
}

\author{
Laboratiore des Signaux et Systèmes (L2S) \\ CNRS, Université Paris-Sud XI (UPS), SUPELEC \\ \{sofiane.braci,remy.boyer,claude.delpha\}@1ss.supelec.fr
}

\begin{abstract}
In this paper, security evaluation of an important watermarking class based on quantization is given. Theoretical developments and practical simulations are used to measure the security level of watermarking techniques. We give the contribution of each observation available to the attacker on the total gathered information about the watermarking secrecy. By leading on watermarking technique weaknesses, we find that using the Quantization Index Modulation (QIM) with continuous secret key is equivalent to using a secret key with two states. The latter is easier to estimate than a continuous key. Then, we propose a secure version of a classical trellis coded quantization watermarking. The security is guaranteed thanks to the trellis path generated from the discrete key and the message. We show that the spread transform can represent a second or alternative security level for watermarking systems. It allows to increase the watermarking security level and to keep the embedded message hard to read for unauthorise user.
\end{abstract}

Index Terms- Security.

\section{INTRODUCTION}

Many of Watermarking techniques claim to be secure just because a secret key is used at the encoder. In reality, they present many weaknesses and the watermarking protection can be broken. The security of a watermarking concerns its capability resist to intentional forgeries. In [1], the authors have proposed the foundations of the watermarking security measure. By leading on Shannon theory on cryptographic security presented in the famous paper "Communication Theory of Secrecy Systems" [2], they evaluate the security of watermarking system by measuring the information leakages about the secret key, used to prevent the unauthorized users reading the embedded message, from the observations available to the attacker. Roughly, the information leakage is calculated thanks to the mutual information between the observation (watermarked copie) and the discrete secret key. When the key is continuous, the information leakage is measured thanks to the fisher information about the secret key from the observation. In both case, we measure the amount of observations (watermarked copies) needed by the attackers to gather enough information and recover the secret key used at the encoder. This paper focuses on the security of an important class of watermarking schemes based on quantization. A security of the distortion compensated (DC)-Quantization Index Modulation (QIM) or DCQIM[3] -equivalent to the Scalar Costa Scheme (SCS)[4]- has been studied in [5] "practically", when a continuous key is used. However, studying the security against specific (practical) attack does not give the limit of the watermarking system security. Luis Pérez-Freire

The authors would like to thank the ESTIVALE and MEDIEVAL projects from ANR (Agence Nationale de la Recherche) for funding. et al. [6] has proposed an evaluation of a DC-QIM security level but by considering implicitly that the secret key is discrete. Then, no one can say if the continuous key guarantee a good protection or not for the QIM watermarking and DC-QIM more generally.

The first contribution of this paper is to show the weakness of the secure QIM (Note that the secure system refers to the version of a watermarking system with a protection which is, generally, given by the secret key) when a continuous secret key is used in the same way as in [5] ( the secret parameter is referred sometimes by "dithering". In this paper, it is called secret key ). We show that using the QIM with continuous key is equivalent to use the QIM with a two states secret key and the attacker estimation becomes easier than with continuous key. The second contribution is an enhancement of the security level for the informed watermarking scheme by using a secure TCQ and a secret spreading direction. To secure the TCQ a trellis path is defined from the message and the secret key. We prove by simulations that the quantity of information leakage from the observations with secure TCQ is less than with secure QIM. For embedding the message over a secret direction, the Spread Transform (ST) [3] is used. Finally, we propose a complete comparative study of security between the secure versions informed watermarking systems based on information theory tools.

Let us first list some notational conventions used in this paper. Vectors are noted in bold font and sets in Calligraphic letters. Data are written in small letters, and random variables in capital letters; $\mathbf{s}[i]$ is the $i^{\text {th }}$ component of vector $\mathbf{s}$.

\section{WATERMARKING SECURITY MEASURE}

As in [1], the watermarking security is provided against an estimations attacks. Then, the cryptographic key must remain secret, no one without authorized persons should be able to break the secrecy. The watermarking security is the ability to make the secrecy recovering difficult for the attacker. In [1] authors assess this difficulties by the size of the observations available to the attackers. They gave theoretical tools from the information theory in order to evaluate the security level. In the following, we summarize the two most important measures of the security level :

1. Shannon's measure : when the secret key is a discrete variable, Shannon [2] used the equivocation in order to measure the efficiency of the secrecy systems. The equivocation gives the rest of the uncertainty after the attacker obtains some information concerning the secrecy of the systems. Formally Shannon's equivocation is given by the conditional entropy $H\left(K \mid X^{N_{0}}\right)$ of the secret parameter conditionally to the available information on the attacker side :

$$
H\left(K \mid X^{N_{0}}\right)=H(K)-I\left(K ; X^{N_{0}}\right),
$$


where $I\left(K ; X^{N_{0}}\right)$ is the mutual information between the secret key $K$-with entropy $H(K)$ - and $N_{0}$ observations copies $X^{N_{0}}$. As in [1], we consider the key used to ciphering the message as the parameter to be concealing from malicious users. The watermarking system security measure criterion is given by the amount of observations $N_{0}$ which tends the equivocation $H\left(K \mid X^{N_{0}}\right)$ to zero. In sequel, the amount of observations available to the attackers $N_{0}$ is the security criterion. However, it is impossible to use the Shannons' measure with a continuous parameter since the entropy does not still a measure of information.

2. Fisher's measure : authors in [1] proposed to use the fisher information in order to measure the security of the watermarking systems instead of the equivocation. It replaces the Shannon measure when the secret parameter is continuous and the joint probability density function, between the available information $O$ and the secret parameter $K$, is derivable.

Due to the derivability constraints of Fishers' measure, the security evaluation of some quantization-based systems with continuous secrete key becomes impossible. For our systems, we shall use the Shannons' measure to evaluate the watermarking security. As it will be shown after, some watermarking systems using the continuous secret are equivalent to watermarking with discrete key.

\subsection{Watermarking security of independent marked copies}

When the secret parameter is discrete, the mutual information between the $N_{0}$ observations and the secret parameter is given by

$$
\begin{array}{r}
I\left(X^{N_{0}} ; K\right)=\sum_{k} \int_{x_{1}} \int_{x_{2}} \ldots \int_{x_{N_{0}}} p\left(x_{1}, x_{2}, \ldots, x_{N_{0}}, k\right) \\
\log \left(\frac{p\left(x_{1}, x_{2}, \ldots, x_{N_{0}}, k\right)}{p\left(x_{1}, x_{2}, \ldots, x_{N_{0}}\right) p(k)}\right),
\end{array}
$$

where $K$ is the random variable which models the secret key and $k[i] \in[-1 / 2,1 / 2], i=1, \ldots, N_{0}$ as in [7].

Since the random variable $X_{i}, i=1, \ldots, N_{0}$ are independent, identically distributed (i.i.d.), such as $x_{i}$ models the $i^{t h}$ marked copy (the $i^{\text {th }}$ observation), its joint density function can be written as

$$
p\left(x_{1}, x_{2}, \ldots, x_{N_{0}}\right)=p\left(x_{1}\right) \cdot p\left(x_{2}\right) \ldots \cdot p\left(x_{N_{0}}\right) .
$$

On the other hand, the marked sample with a fixed secret key word is formulated as follow :

$$
x_{i}=s_{i}+w_{i, k}, i=1, \ldots, N_{0},
$$

such as $w_{i, k}$ is the watermark of the $i^{t h}$ observation for a secret key word $k$. We note that for a fixed key word $k$ the marked samples $x_{i}, i=1, \ldots, N_{0}$ remain independent, then

$$
p\left(x_{1}, x_{2}, \ldots, x_{N_{0}} \mid k\right)=p\left(x_{1} \mid k\right) \cdot p\left(x_{2} \mid k\right) \ldots \cdot p\left(x_{1} \mid k\right),
$$

with a marginalization, we obtain

$$
\begin{gathered}
I\left(X^{N_{0}} ; K\right)=\underbrace{\sum_{k} \int_{x_{1}} p\left(x_{1}, k\right) \log \left[\frac{p\left(x_{1} \mid k\right)}{p\left(x_{1}\right)}\right]}_{I\left(X_{1} ; K\right)} \\
+\underbrace{\sum_{k} \int_{x_{2}} p\left(x_{2}, k\right) \log \left[\frac{p\left(x_{2} \mid k\right)}{p\left(x_{2}\right)}\right]}_{I\left(X_{2} ; K\right)} \\
+\quad \ldots+\underbrace{\sum_{k} \int_{x_{N_{0}}} p\left(x_{N_{0}}, k\right) \log \left[\frac{p\left(x_{N_{0}} \mid k\right)}{p\left(x_{N_{0}}\right)}\right]}_{I\left(X_{N_{0}} ; K\right)} .
\end{gathered}
$$

Then, the information leakages from $N_{0}$ observations, in the case of informed watermarking schemes, is the sum of the information leakages from each independent observation, i.e,

$$
I\left(X^{N_{0}} ; K\right)=\sum_{i=1}^{N_{0}} I\left(X_{i} ; K\right) .
$$

When the equivocation is equal to zero, it means that there is no secrecy. If the same informed watermark schemes is used for all the observations and from Eqn.1, the amount of observations needed for completely estimate the secret key becomes

$$
N_{0}=\frac{H(K)}{I(X ; K)} .
$$

In the sequel, we use Eqn.7 to compute the security level of the watermarking system.

Note that up until now, there have been no works on the security evaluation of the QIM, and more generally, the SCS watermarking with a continuous secret key. It is due to the continuity of the secret key. In addition, it is impossible to measure the Fisher information. In the next section, we give a detailed analysis of the QIM security and propose a new secure system based on TCQ watermarking.

\section{QUANTIZATION-BASED WATERMARKING SCHEMES AND SECURITY ANALYSIS}

\subsection{The Quantization Index Modulation}

QIM [3] is a system based on scalar quantization of host signal. It is a particular case of the SCS also called DC-QIM. Let us consider a binary message $\mathbf{m}$ to be embedded and a host signal $\mathbf{s}$. In the QIM watermarking system, two codebooks are defined using two shifted uniform scalar quantizers :

$$
\begin{aligned}
& \mathcal{U}_{0}[i]=\{n \Delta+\mathbf{k}[i] \Delta, n \in \mathcal{Z}\} \text { and } \\
& \mathcal{U}_{1}[i]=\left\{n \Delta+\mathbf{k}[i] \Delta+\frac{\Delta}{2}, n \in \mathcal{Z}\right\}
\end{aligned}
$$

where $\Delta$ is a quantization step, $\mathbf{k}[i]$ represents $i^{\text {th }}$ component of a continuous secret key -given by the vector $\mathbf{k}-$, such as $\mathbf{k}[i] \in$ $[-1 / 2,1 / 2]$, and $\mathcal{Z}$ is the set of integers. According to the bit $\mathbf{m}[i]$ to be embedded, one of the two codebooks is selected and the nearest codeword $\mathbf{u}^{\star}[i]$ to $\mathbf{s}[i]$ is chosen. The marked signal is given by : $\mathbf{x}[i]=\mathbf{u}^{\star}[i]$. We can use the embedding formulation proposed in [4] and fixe the Costas' parameter $\alpha$ to one which mean that the embedder consider the channel noiseless (see [4] and [3] for details). In order to extract the message, the decoder computes some decision criterion denoted $\mathbf{y}$, given by the following expression [4] :

$$
\mathbf{y}=Q_{\Delta}(\mathbf{x}-\mathbf{k} \Delta)-(\mathbf{x}-\mathbf{k} \Delta)
$$

where $\mathbf{x}$ is the received signal without channel distortion and $Q_{\Delta}($. is the scalar quantizer with step $\Delta$.

When there is no noise the bit message is equal to 1 if $|\mathbf{y}[i]|=\frac{\Delta}{2}$, or to 0 if $\mathbf{y}[i]=0$. When the channel is noisy, we proceeds as following :

$$
m=\left\{\begin{array}{l}
1 \text { if }|\mathbf{y}[i]|>\Delta / 4 \\
0 \text { if }|\mathbf{y}[i]| \leq \Delta / 4
\end{array}\right.
$$

Unfortunately, the attacker has a noiseless watermarked copy and has access to all embedding parameter except the secret key (Kerckhoffs' principle). Thus, it is possible for the attacker to consider the secret key as noise. If someone try to estimate the message without using the exact key, the decision criterion $\mathbf{y}[i]$ for the $i^{t h}$ marked sample is 


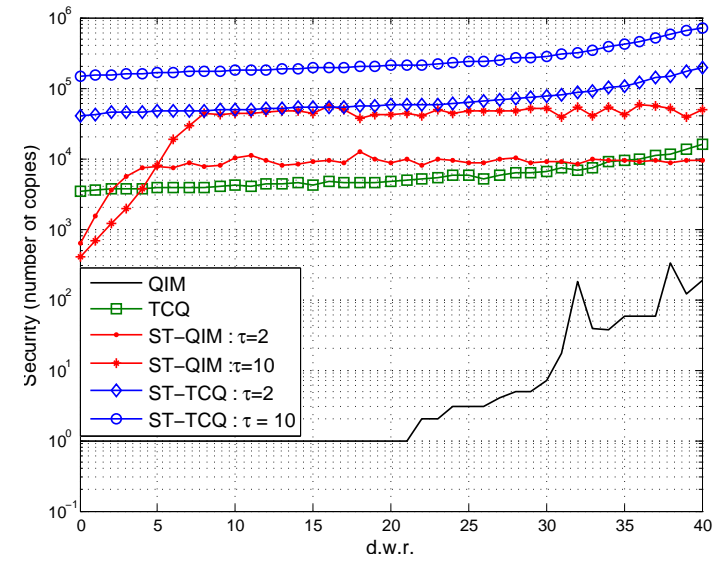

Fig. 1. The security level measured by the amount of observations required to estimate the secret key as function of the document to watermark ratio (d.w.r.) for QIM, TCQ, ST-QIM and ST-TCQ.

computed "blindly" (the decision criterion is computed as if there is no key) as

$$
\begin{aligned}
\mathbf{y}[i] & =Q_{\Delta}(\mathbf{x}[i])-\mathbf{x}[i] \\
& =Q_{\Delta}\left(n \Delta+\mathbf{k}[i] \Delta+\mathbf{m}[i] \frac{\Delta}{2}\right) \\
& -\left(n \Delta+\mathbf{k}[i] \Delta+\mathbf{m}[i] \frac{\Delta}{2}\right), \quad n \in \mathcal{Z} .
\end{aligned}
$$

Note that Eqn.11 results from Eqn.8 (when the encoder uses a secret key k) and Eqn.9 (without key $\mathbf{k}$, the attacker does not know the secret key) when the signal is noiseless (the attacker receives a noisless copies [8]).

In the following, our purpose is to show the weakness of the secure version of QIM when a continuous secret key is used. A simple development shows that it is possible to perfectly decode the embedded message $\mathbf{m}$, by using Eqn.10, if $|\mathbf{k}[i]| \leq 1 / 4$. A wrong message decoding happen only if $|\mathbf{k}[i]|>1 / 4$. Thus, the attacker computes a new decision criterion $\mathbf{y}^{\prime}[i]$ as following :

$$
\mathbf{y}^{\prime}[i]=\left\{\begin{array}{l}
Q_{\Delta}(\mathbf{x}[i])-\mathbf{x}[i] \text { if }|\mathbf{k}[i]| \leq 1 / 4 \\
Q_{\Delta}(\mathbf{x}[i]-\Delta / 4)-(\mathbf{x}[i]-\Delta / 4) \text { if }|\mathbf{k}[i]|>1 / 4
\end{array}\right.
$$

then, he use Eqn.10 to decode the correct message.

In other words, the attacker has no need to know the exact value of the secret key. All that he/she needs is to estimate if the absolute value of the $i^{t h}$ secret key $|\mathbf{k}[i]|$ is above or below the threshold $1 / 4$. If the absolute value of the $i^{t h}$ secret key value is under the threshold $1 / 4$, the attacker knows that there is no need to use a key. However, if $|\mathbf{k}[i]|>1 / 4$, the attacker just subtract $\Delta / 4$ from the $i^{t h}$ received sample value $\mathbf{x}[i]$ to recover the message. Thus, the attacker is able to reduce the secret key alphabets from the infinity -due to the continuity of the secret key- to only two states of the secret key : $|\mathbf{k}[i]| \leq 1 / 4$ or $|\mathbf{k}[i]|>1 / 4$.

We compute the bit error rate at the decoder, when an estimation of the key (above or below 1/4) is used according to the method described before. Of course, we use Eqn.12 to decode the message $\mathbf{m}$. We find that the bit error rate, in the attacker side, is under $10^{-6}$. The same arguments can be applied for the SCS with continuous key proposed in [4] (a security weakness by reducing the secret key alphabets). However, the Costas'parameter $\alpha$ must be close to 1 in order to decode $\mathbf{m}$ with a limited error (error under $1 \%$ ).

\subsection{The Trellis Coded Quantization (TCQ) scheme}

In order to prevent the key estimation by using the weakness on the QIM decoder, we use the TCQ watermarking since the decoder give the path corresponding to the whole message. Then, the attacker has to estimate all secrete keys in one time.

Let us consider a trellis defined by a transition function : $\mathcal{E} \times$ $\{0,1\} \longrightarrow \mathcal{E}, \operatorname{tr}:(\mathbf{e}[i], \mathbf{m}[i]) \longmapsto \mathbf{e}[i+1]$, with $\mathcal{E}=\left\{0,1, \ldots, 2^{r-1}\right\}$ groups of possible states, where $r$ is an integer such as $r>1$, and $i$ is the index of the current transition. Contrary to the QIM (see Eqn.8), the shifting $\mathbf{d}$ becomes a function of the current state and the embedded symbol :

$$
\begin{aligned}
\mathcal{E} \times\{0,1\} & \longrightarrow[-\Delta / 2,+\Delta / 2], \\
f:(\mathbf{e}[i], \mathbf{m}[i]) & \longmapsto \mathbf{d}[i] .
\end{aligned}
$$

In this watermarking, the codebooks are defined by :

$$
\mathcal{U}_{\mathbf{m}}[i]=\{n \Delta+f(\mathbf{e}[i], \mathbf{m}[i]), n \in \mathcal{Z}\},
$$

and the closest codeword $\mathbf{u}^{\star} \in \mathcal{U}_{\mathbf{m}}$ to $\mathbf{s}$ is chosen using the Viterbi algorithm [9] in order to be sure that the obtained codeword belongs to $\mathcal{U}_{\mathbf{m}}$. The marked signal is given by : $\mathbf{x}=\mathbf{u}^{\star}$.

To extract the embedded message, we have to apply the Viterbi algorithm in order to retrieve the path which corresponds to the watermarked signal.

\subsubsection{Secure $T C Q$}

In [10], authors studied the classical TCQ watermarking security, where system parameters are kept secret and no ciphering message key is used. In our secure TCQ, the main idea is not only to cipher the message but also the path. Thus, the marked signal is perceptually the same as the non-secure, because by changing the path we only change the quantization codebook. However, it prevents all unauthorized users to extract the message. At the encoder, after quantizing the host-signal, we shift randomly each obtained codeword to a new one taking from another sub-codebook. Then, according to the secret key we choose a codebook which is different from the one used for the transmitted message. It is hard for an attacker to extract the correct message, since he has to test all possible trellis paths. At the decoder side, by using the secret key we put the received message in the right codebook -and also in the right trellis path- and then we extract the corresponding message.

In order to evaluate and compare the security level of the studied watermarking systems, we use Eqn.7 to calculate the amount of copies needed to entirely estimate the secret key and the simulation result are given in Fig.1. We note the enhancement of the security level, measured by the amount of observations, when the secure TCQ is used. It is clear that it much harder for the attacker to estimate the key in the case of secure TCQ than the secure QIM.

In [2] Shannon showed that the protection efficiency depends on the secret key alphabet and the secret key length. However, the maximum secret key alphabet size for the secure TCQ is the state number and the key length cannot exceed that of the host signal. Then, it is impossible to go up some limit of the TCQ security level.

\section{SPREAD TRANSFORM : ALTERNATIVE OR/AND SECOND SECURITY LAYER}

It is possible to increase the security of the watermarking systems by adding a new protection layer. On the other hand, using the ST allows to choose a secret direction for the watermark insertion without interfering with the watermarking security system. Thus, we can use the ST as an alternative or second protection layer for the watermarking. Chen and Wornel in paper [3] have introduced an 


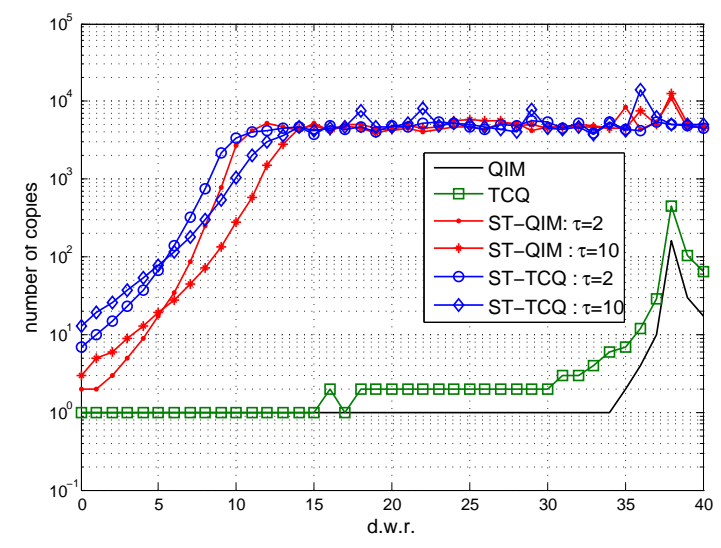

Fig. 2. The amount of observations required to estimate the hiding message as function of the document to watermark ratio (d.w.r.) for QIM, TCQ, ST-QIM and ST-TCQ.

efficient watermarking scheme which allows to spread the message on several host samples. The spreading of the host signal, denoted by $\mathbf{s}=\left[s_{0}, \ldots, s_{M-1}\right]$, is given by $\mathbf{s}^{S T}[l]=\sum_{i=\tau l}^{\tau l+\tau-1} \mathbf{s}[i] \mathbf{t}[i]$, where $\tau \in \mathcal{N}^{\star}$ is the spreading factor. The above operation is in fact a projection along direction $\mathbf{t}$, where $\mathbf{t}$ is a unitary vector. Next, the inverse transformation is applied to the watermarked signal according to $\mathbf{x}^{S T}=\mathbf{s}^{S T}+\mathbf{w}^{S T}$-where $\mathbf{w}^{S T}$ is the watermark in the transformed domain- and we have [4] : $\quad \mathbf{x}=\mathbf{s}+\mathbf{w}^{S T} \cdot \mathbf{t}$.

There is two interesting combinations with the ST. The first one is the ST-QIM. Proposed by Eggers [4], he showed that the ST improves the robustness against the AWGN attack. In our previous work [11], we showed the good steganographic performance of the ST-QIM. From Fig.1, we note that the ST-QIM has a better security level than the QIM thanks to using a secret direction. The latter improvement is obtained thanks to the high dimensional space from which the ST spreading direction is taken. Then the ST can be seen as second protection which improves the watermarking system security.

Another interesting combination is the ST-TCQ, we proposed it in [12] where we demonstrated the good performance of that system. Fig.1 shows that ST-TCQ allows the best security level for the watermarking thanks to the secret direction and the secure TCQ.

On the other hand, "Security refers to the inability by unauthorized users to have access to the raw watermarking channel". Even if it is not an exhaustive analysis, we have studied the attacker access to the channel protection and his ability to extract the watermarking after breaking the security system through Fig.2. The results given in Fig. 2 are obtained by measuring the amount of copies $N_{0}$ needed to recover the embedded message (it is simple to prove that : $N_{0}=H(M) / I(X ; M)$ in the same way as we prove Eqn.1). Generally, it is supposed that if the attacker estimates the secret key, he can extract any embedding mark and use it to produce authorized copies. However, from Fig. 2 we note that the systems marked along a secret direction (thanks to the ST) make the watermark extraction difficult for the attacker. In other words, the amount of copies needed to extract the message, after estimating the secret key, when we use the ST is bigger than when it is not used.

\section{CONCLUSIONS}

The QIM is usually presented as a secure system when we use a continuous key. Theoretically, the continuous characters of the key prevent most of estimation attacks. We have shown in this work that it is possible to reduce the estimation of the QIM continuous secret key to an estimation of a discrete key with only two states. Then, a new secure version of the TCQ watermarking systems is proposed where a secret key is used to give a wrong path to all unauthorised users. The secret key words are equal to the number of the trellis states. The larger the trellis state number, the more important is the security level of the watermarking system. In order to enhance the security, we have evaluated the assignment giving by the use of a secret spreading direction. Then, we have compared the security performances of the combination of the ST with QIM and TCQ. We have found that the ST-TCQ is the most secure system.

From our previous works on a stego-security, we note that there is a correlation between the stego-security and the watermarking security. For the future works, we shall study more precisely this relationship and verify theoretically if the spread transform guarantee the security against a collusion attacks.

\section{ACKNOWLEDGMENT}

The authors would like to thank Professor Pierre Duhamel for his help and collaboration to this paper.

\section{REFERENCES}

[1] F. Cayre, C. Fontaine, and T. Furon. Watermarking security : Theory and practice. IEEE Transactions on Signal Processing, 53(10) :3976-3987, 2005. numéro spécial "Supplement on Secure Media III".

[2] C. Shannon. Communication theory of secrecy systems. Bell System Technical Journal, 28 :656-715, 1949.

[3] B. Chen and G. W. Wornell. Quantization index modulation : a class of provably good methods for digital watermarking and information embedding. IEEE Trans. on Information Theory, 47 :1423-1443, May 2001.

[4] J. J. Eggers, R. Bauml, R. Tzchoppe, and B. Girod. Scalar costa scheme for information embedding. IEEE Trans. on Signal Processing, April 2003.

[5] Patrick Bas and Jarmo Hurri. Security of dm quantization watermarking schemes : A practical study for digital images. In $I W D W$, Lecture Notes in Computer Science, pages 186-200. Springer, 2005.

[6] S. Voloshynovskiy L. Pérez-Freire, F. Pérez-González. Revealing the true achievable rates of scalar costa scheme. In 6th International Workshop on Multimedia Signal Processing, pages 203-206, Siena, Italy, 2004. IEEE.

[7] P. Guillon, T. Furon, and P. Duhamel. Applied public-key steganography. In Proc. SPIE, San Jose, CA, 2002.

[8] I. J. Cox, M. L. Miller, J. A. Bloom, J. Fridrich, and T. Kalker. Digital watermarking and steganography. Morgan Kaufmann, second edition, 2008.

[9] G. D. Forney Jr. The viterbi algorithm. Proc. IEEE, 61 :268278, March 1973.

[10] P. Bas and G. Doërr. Practical security analysis of dirty paper trellis watermarking. In LNCS, Information Hiding, SaintMalo, France, 2007.

[11] S. Braci, C. Delpha, R. Boyer, and G. Le Guelvouit. Informed stego-systems in active warden context : Statistical undetectability and capacity. IEEE Proc. MMSP, October 2008.

[12] S. Braci, R. Boyer, and C. Delpha. On the tradeoff between security and robustness of the trellis coded quantization scheme. IEEE International Conference on Accoustics, Speech and Signal Processing (ICASSP), April 2008. 